\title{
ANCA-Positive Patients: The Influence of PR3 and MPO Antibodies on Survival Rate and The Association with Clinical and Laboratory Characteristics
}

\author{
J.C. Drooger ${ }^{*}$, A. Dees and A.J.G. Swaak \\ Departments of Internal Medicine and Rheumatology, Ikazia Hospital, Postbus 5009, 3008 AA, Rotterdam, \\ The Netherlands
}

\begin{abstract}
Objectives: To compare the survival rate, and the clinical and laboratory characteristics in patients, characterized by the presence of certain anti-neutrophil cytoplasmic auto-antibodies (ANCAs).

Methods: In a retrospective observational study, we analyzed the data of all patients with a positive ANCA test between 1995 and 2005 at our hospital. Based on serology patients were divided in three subgroups (ANCA-Proteinase 3 (PR3), ANCA-Myeloperoxidase (MPO) and atypical ANCA), irrespective of the diagnosis. Patient survival was compared by Kaplan Meier survival analysis. Differences in clinical and laboratory characteristics between the groups of specific ANCAs were determined.

Results: Fifty-four ANCA-positive patients were analyzed. Eighteen of these patients were ANCA-PR3-positive, 17 were ANCA-MPO-positive and 19 had a atypical ANCA. A random control group was created of matched ANCA negative patients. Average follow-up time was 52 months. The calculated five year survival rate in respectively the ANCA-PR3positive group, the ANCA-MPO-positive group, the atypical ANCA group and the ANCA-negative group was 45\%, $81 \%$, $90 \%$ and $100 \%$. ( $P=0.012$, Wilcoxon test). A higher mean leukocyte count, a higher mean erythrocyte sedimentation rate and more fever was observed in the ANCA-PR3-positive group compared to the ANCA-MPO-positive group.

Conclusions: A remarkable lower survival rate was observed in ANCA-PR3-positive patients compared to ANCA-MPOpositive patients. We also demonstrated that patients characterized by the presence of a defined ANCA differ in clinical and laboratory characteristics.
\end{abstract}

Keywords: ANCA, anti-myeloperoxidase (MPO), anti-proteinase3 (PR3), survival, prognostic value.

\section{INTRODUCTION}

Anti-neutrophil cytoplasmic auto-antibodies (ANCAs) are important serologic markers for vasculitis. ANCAassociated diseases comprise a heterogeneous group of diseases that are generally considered uncommon, containing not only vasculitides, but also a variety of other (systemic) diseases $[1,2]$.

ANCAs are directed against several myeloid enzymes. By indirect immunofluorescence microscopy on neutrophils two major categories can be distinguished: cytoplasmic ANCA and perinuclear ANCA. By ELISA testing cytoplasmic ANCA nearly always reacts with proteinase 3 (ANCAPR3). The major perinuclear ANCA target is myeloperoxidase (ANCA-MPO). In atypical ANCAs the immunofluorescence test is positive (mostly perinuclear ANCA), but the ELISA for both ANCA-MPO and ANCA-PR3 is negative.

Data from recent studies seem to confirm the long- disputed pathogenic role of ANCAs [3, 4]. ANCA-PR3-positivity is predominantly found in patients with Wegener's

*Address correspondence to this author at the Departments of Internal Medicine and Rheumatology, Ikazia Hospital, Postbus 5009, 3008 AA, Rotterdam, The Netherlands; Tel: +31 10 2975000; Fax: +31 10 2975400; E-mail: jcdrooger@kliksafe.nl granulomatosis and microscopic polyangiitis. ANCA-MPOpositivity is also found in patients with microscopic polyangiitis as well as in patients with other vasculitides and auto-immune conditions [1]. Atypical ANCAs are seen in a wide variety of medical conditions.

Most prospective studies compare characteristics of ANCA subgroups within a certain diagnosis and with strict entry criteria. Few studies used the ANCA serology as entry point for analysis. Former studies showed that in patient with Wegener's granulomatosis ANCA-PR3-positive patients have more relapses and a shorter survival period compared to ANCA-MPO-positive patients $[5,6]$. In a cohort of patients with Wegener's granulomatosis or microscopic polyangiitis, a poorer prognosis was found in patients with ANCA-PR3 positivity compared to patients with ANCA-MPO positivity $[7,8]$.

The objective of this study was to compare the survival rate, and the clinical and laboratory characteristics in patients, characterized by the presence of ANCA-MPO and ANCA-PR3, irrespective of the diagnosis. All new ANCApositive patients over a 10-year period at our hospital, were analyzed. In contrast to former studies, the different serologic subgroups were compared irrespective of the diagnosis and without strict entry criteria. ANCA testing was performed in cases with clinical suspicion of a systemic disease. 
Firstly the survival rates of the different serologic subgroups were investigated. Secondly differences in clinical and laboratory characteristics were determined. We tried to formulate which combination of clinical parameters correlated with the presence of a positive ANCA.

\section{MATERIAL AND METHODOLOGY}

A retrospective study was performed at Ikazia Hospital, a general teaching hospital in Rotterdam, the Netherlands. All patients, who had been tested for the first time for ANCA serology between 1995 and 2005, were identified by Sanquin (central laboratory, Amsterdam). Detection of ANCA by immunofluorescence [9] and testing of ANCA target antigens by ELISA [10] were performed according to standardized European guidelines. There were no exclusion criteria.

Fifty-four ANCA-positive patients were analyzed. Eighteen patients were ANCA-PR3 positive, 17 were ANCAMPO positive and 19 had an atypical ANCA. A random control group was created of ANCA negative patients, matched for age and gender. Starting date of study entry was the date of the first ANCA serology.

Clinical, laboratory and survival data were collected retrospectively from the patients' charts. Treatment was not uniform because of the wide variety of diagnoses. Part of the patients received immunosuppressive drugs. Analyses were performed with all ANCA positive patients and a matched sample of ANCA negative patients.

Baseline characteristics of the different groups were compared. We constructed Kaplan-Meier survival curves for the different ANCA subgroups. Starting date for the curves was the date on which ANCA serology was obtained. The generalized Wilcoxon (Breslow) test was performed to test the equality of survival distributions for the different subgroups.

The relevant clinical and laboratory differences between the groups were analyzed.

Differences between means were tested using the Student's t-test. The chi-square test was used to compare categorical data. All tests were two-tailed and P-values of $<0.05$ were considered significant. Analyses were performed with SPSS version 14.0 (SPSS Inc., Chicago, IL, USA).

\section{RESULTS}

Fifty-four patients had a positive ANCA immunofluorescence test. These patients were divided into three subgroups. A random control group of ANCA-negative patients was created. The indication to test for ANCA was in all cases a clinical suspicion of the presence of an auto-immune disease.

Mean follow-up time was 52 months. Mean follow-up time in the atypical ANCA group was 72 months (significantly higher). Mean age was 56 years. There were no significant differences in mean age and sex distribution between the groups. Fifty-seven percent of the patients underwent tissue biopsy. The diagnoses were classified according tot the international classification of diseases (ICD-10). Diagnosis in the ANCA-PR3-positive group was mostly Wegener's granulomatosis (72\%). Other diagnoses in the ANCA-PR3positive group were: Churg-Strauss, sarcoidosis, idiopathic pulmonary fibrosis, polyarteritis nodosa and hypersensitivity angiitis. In the ANCA-MPO-positive group one patient was

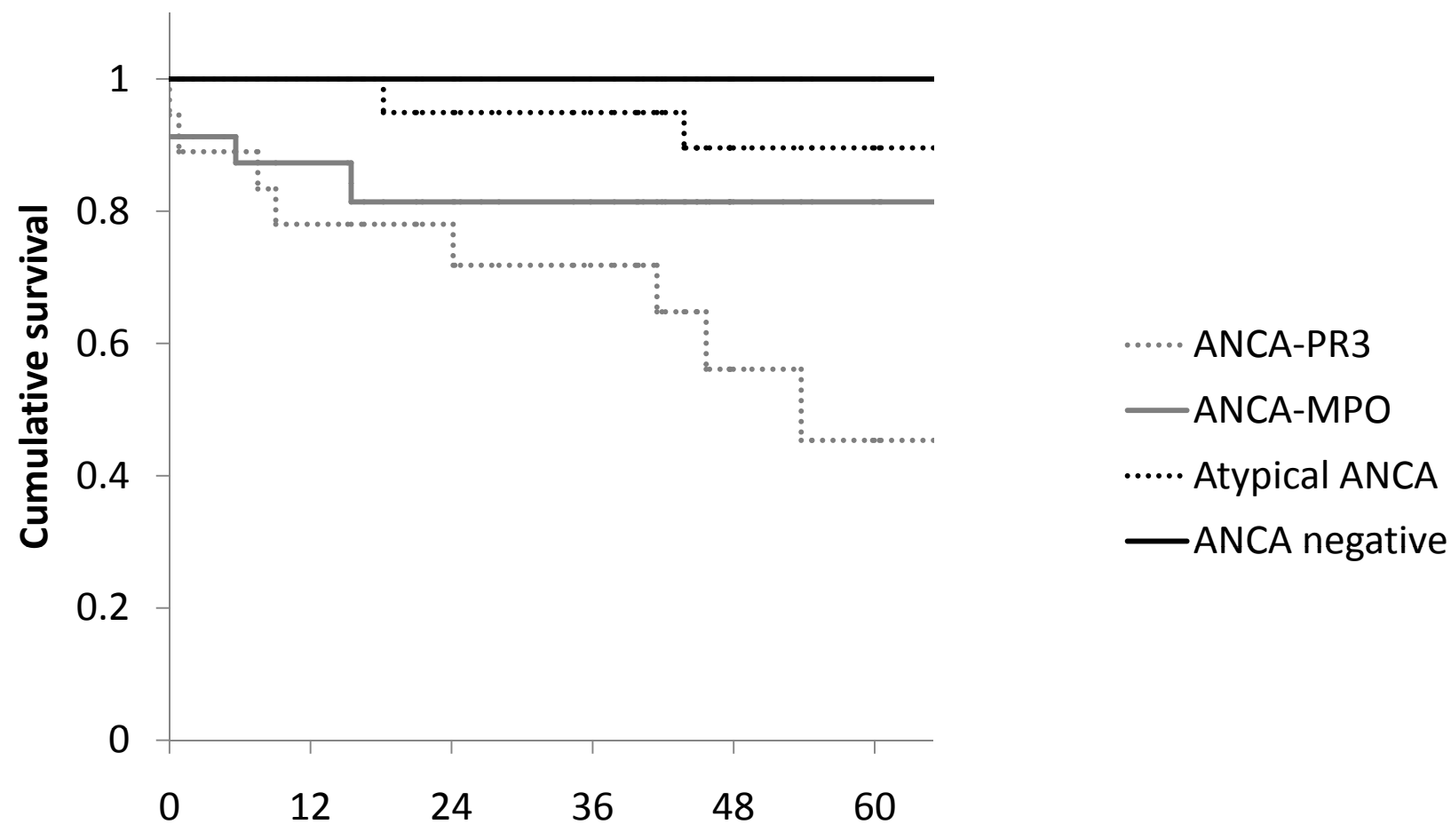

Follow up (months)

Fig. (1). Calculated five year survival in respectively the ANCA-PR3-positive group, the ANCA-MPO-positive group, the atypical ANCA group and the ANCA-negative group. The curves differ significantly ( $\mathrm{P}=0.012$, Wilcoxon test). 
diagnosed with Wegener's granulomatosis. Other diagnoses in the ANCA-MPO-positive group were: systemic lupus erythematosus, seronegative rheumatoid arthritis, idiopathic pulmonary fibrosis, microscopic polyangiitis, giant cell arteritis, polymyalgia rheumatica and Sjögren's syndrome. Diagnoses in the atypical ANCA group were among others: mixed connective tissue disease, polymyositis, systemic sclerosis and Crohn's disease.

The calculated five year survival rate in respectively the ANCA-PR3-positive group, the ANCA-MPO-positive group, the atypical ANCA group and the ANCA-negative group was $45 \%, 81 \%, 90 \%$ and $100 \%$ (Fig. 1). The generalized Wilcoxon (Breslow) test was performed to test the equality of survival distributions for the different subgroups. The survival curves differed significantly $(\mathrm{P}=0.012)$.

Table 1 shows the differences in laboratory and clinical characteristics, comparing the ANCA-PR3-positive group with the ANCA-MPO-positive group. The laboratory results showed significant differences in mean leukocyte count and erythrocyte sedimentation rate (ESR) between the subgroups. The incidence of erytrhocyturia and proteinuria was higher in the ANCA-PR3-positive group. No significant differences were seen in hemoglobin level and platelet count. Ear-nose-throat symptoms and fever were observed more often in the ANCA-PR3-positive group. No significant differences were seen in the incidence of dyspnoe and weight loss.

Table 1. Differences in Laboratory and Clinical Characteristics, Comparing the ANCA-PR3-Positive Group with the ANCA-MPO-Positive Group

\begin{tabular}{|l|c|c|c|}
\hline & ANCA-PR3+ & ANCA-MPO+ & P-Value \\
\hline \hline Leukocyte count (*109/1) & 13.4 & 8.7 & 0.001 \\
\hline Hemoglobin (mmol/1) & 6.9 & 7.6 & 0.144 \\
\hline Platelet count (*109/l) & 371 & 305 & 0.090 \\
\hline Mean ESR (mm/hr) & 86 & 58 & 0.010 \\
\hline Erytrocyturia (\%) & 81 & 38 & 0.012 \\
\hline Proteinuria (\%) & 88 & 50 & 0.022 \\
\hline Dyspnoe (\%) & 39 & 41 & 0.890 \\
\hline Fever (\%) & 56 & 12 & 0.006 \\
\hline Weight loss (\%) & 56 & 41 & 0.395 \\
\hline
\end{tabular}

The conclusion can be drawn that the triad elevated ESR, erythrocyturia and malaise might be useful when considering ANCA testing. The combination of these three characteristics was seen in 73 percent of the patients in the ANCAPR3-positive group. In the ANCA-negative group no patients had the combination of these characteristics.

\section{DISCUSSION}

A remarkable difference in survival rate was observed in patients characterized by the presence of a defined ANCA. ANCA-PR3-positive patients showed a poor prognosis compared to ANCA-MPO-positive patients.
We could demonstrate that patients characterized by the presence of a defined ANCA differ not only in diagnoses, but also in a variety of clinical and laboratory characteristics. ANCA-PR3-positive patients demonstrated a more extensive disease state. The triad elevated ESR, erythrocyturia and malaise was observed more often in ANCA positive patients and can therefore be used as a clinical prediction estimate, when considering obtaining ANCA serology.

Previous studies have shown a lower survival rate in ANCA-PR3 patients [5, 7], compared to ANCA-MPO patients. Most of these studies analyzed the value of specific ANCAs within specific diagnostic subgroups, while we included all ANCA positive patients irrespective of the diagnosis. As far as we know there is only one other study, which used the serology as entry point for analysis [11]. In that study no difference in survival between ANCA-PR3 and ANCA-MPO was found. The survival rate, we found in ANCA-PR3 positive patients is lower then reported on large cohorts of PR3-ANCA positive patients with vasculitis [1, 13]. We included all PR3 positive patients irrespective of the diagnoses, while earlier studies had strict entry criteria and included only patients with a biopsy-proven diagnosis. So the survival rate in earlier studies might be overestimated because patients with a worse prognosis (e.g. elderly) were excluded.

We found clear differences in laboratory and clinical parameters between the different groups. ANCA-PR3 patients demonstrated a more aggressive disease state. They have more extensive inflammation parameters. This confirms the findings of previous studies $[2,6,12]$.

The three subgroups (ANCA-PR3, ANCA-MPO and atypical ANCA) were equally distributed in our population. ANCA testing is often done with a low pre-test probability of systemic small vessel vascultis. A more critical approach in obtaining ANCA tests will increase the diagnostic value of ANCA-testing [8, 14]. The investigated clinical and laboratory characteristics and the proposed triad could help when ANCA testing is considered. In our opinion ANCA testing should only be performed when there is a fair clinical suspicion of an ANCA-associated vasculitis.

The numbers in both groups are small. Because of lack of power, we could not exclude that the differences in survival rate between ANCA-PR3 and ANCA-MPO-positive patients are partly based on differences in diagnoses in the groups. The small numbers in the subgroups underscore the fact that ANCA-related diseases are seldom seen in general hospitals. For example, when taking into account the incidence of Wegener's granulomatosis and the adherence population of Ikazia Hospital, we expected one or two new patients per year. The low incidence of these diseases is an argument to enroll all new patients in prospective studies.

In clinical practice ANCA serology is mainly used as a diagnostic tool, in combination with tissue examination. Next to the diagnostic significance of the ANCA serology, this retrospective study demonstrates the prognostic value of the specific ANCA-positivity.

Because of the low incidence of ANCA-associated diseases, to define the clinical and prognostic value of (specific) ANCA-serology more extensive (probably multi-center) and prospective research is necessary. 


\section{REFERENCES}

[1] Schönermarck U, Lamprecht P, Csernok E, Gross WL. Prevalence and spectrum of rheumatic diseases associated with proteinase 3antineutrophil cytoplasmic antibodies (ANCA) and myeloperoxidase-ANCA. Rheumatology 2001; 40: 179-84.

[2] Goldschmeding R, Cohen Tervaert JW, Gans ROB, et al. Different immunological specificities and disease associations of c-ANCA and p-ANCA. Neth J Med 1990; 36: 114-16.

[3] Bosch X, Guilaber A, Font J. Antineutrophil cytoplasmic antibodies. Lancet 2006; 368: 404-41.

[4] Kallenberg CGM. Antineutrophil cytoplasmic autoantibody-associated small-vessel vasculitis. Curr Opin Rheumatol 2007; 19: 17-24.

[5] Weidner S, Geuss S, Hafezi-Rachti S, Wonka A, Rupprecht HD. ANCA-associated vasculitis with renal involvement: an outcome analysis. Nephrol Dial Transplant 2004; 19: 1403-11.

[6] Franssen CFM, Stegeman CA, Kallenberg GCM, et al. Antiproteinase 3-and antimyeloperoxidase-associated vasculitis. Kidney Int 2000; 57: 2195-206.

[7] Westman KWA, Bygren PG, Olsson H, Ranstam J, Wieslander J. Relapse rate, renal survival, and cancer morbidity in patients with Wegener's granulomatosis or microscopic polyangiitis with renal involvement. J Am Soc Nephrol 1998; 9: 842-52

[8] Westman KWA, Selga D, Isberg P, Bladström A, Olsson H. High proteinase 3-anti-neutrophil cytoplasmic antibody (ANCA) level measured by the capture enzyme-linked immunosorbent assay method is associated with decreased patiënt survival in ANCA- associated vasculitis with renal involvement. J am Soc Nephrol 2003; 14: 2926-33.

[9] Wijk A, Rasmussen N, Wieslander J. Methods to detect autoantibodies to neutrophilic granulocytes. In: van Venrooij WJ, Maini RN, Eds. Manual of biological markers of disease. Dordrecht: Kluwer Academic Publishers 1993; vol. A9: pp. 1-14.

[10] Hagen EC, Andrassy K, Csernok E, et al. Development and standardization of solid phase assays for the detection of antineutrophil cytoplasmic antibodies (ANCA). A report on the second phase of an international cooperative study on the standardization of ANCA assays. J Immunol Methods 1996; 196(1): 1-15.

[11] Franssen CFM, Gans ROB, Arends B, et al. Differences between anti-myeloperoxidase-and anti-proteinase 3-associated renal diseases. Kidney Int 1995; 47: 193-99.

[12] Franssen C, Gans R, Kallenberg C, Hageluken C, Hoorntje S. Disease spectrum of patients with antineutrophil cytoplasmic autoantibodies of defined specificity: distinct differences between patients with anti-proteinase 3 and anti-myeloperoxidase autoantibodies. J Intern Med 1998; 244: 209-16.

[13] Slot MC, Cohen Tervaert JW, Franssen CFM, Stegeman CA. Renal survival and prognostic factors in patients with PR3-ANCA associated vasculitis with renal involvement. Kidney Int 2003; 63: 67077.

[14] Mandl LA, Solomon DH, Smith EL, Lew RA, Katz JN, Shmerling $\mathrm{RH}$. Using antineutrophil cytoplasmic antibody testing to diagnose vasculitis. can test-ordering guidelines improve diagnostic accuracy? Arch Intern Med 2002; 162: 1509-14.

(c) Drooger et al.; Licensee Bentham Open.

This is an open access article licensed under the terms of the Creative Commons Attribution Non-Commercial License (http://creativecommons.org/licenses/by$\mathrm{nc} / 3.0 /$ ) which permits unrestricted, non-commercial use, distribution and reproduction in any medium, provided the work is properly cited. 